\title{
Regularity of Fetal HRV Changes in an In-vivo Sheep Model of Labor
}

\author{
Massimo W. Rivolta ${ }^{1}$, Md Aktaruzzaman ${ }^{1}$, Tamara Stampalija ${ }^{2}$, Daniela Casati ${ }^{3}$, Martin G. Frasch $^{4}$, \\ Enrico Ferrazzi ${ }^{3}$, Roberto Sassi ${ }^{1}$ \\ ${ }^{1}$ Dipartimento di Informatica, Università degli Studi di Milano, Milan, Italy \\ ${ }^{2}$ Unit of Prenatal Diag., Inst. for Maternal and Child Health, IRCCS Burlo Garofolo, Trieste, Italy \\ ${ }^{3}$ Dept. of Woman, Mother and Neonate, Buzzi Children's Hospital, Biomedical and Clinical Sciences \\ School of Medicine, University of Milan, Milan, Italy \\ ${ }^{4}$ Department of Obstetrics and Gynecology, Universitv of Washington. Seattle. WA. USA
}

\begin{abstract}
Labor exposes the fetus to repetitive transient hypoxic stress. We assessed whether such events modify the regularity of the fetal inter-beat interval series (fRR), using an in-vivo near-term sheep model, by means of entropy measures. Umbilical cord occlusions (UCO), from partial to complete, were applied to 7 near-term pregnant sheep. Fetal blood samples were collected at intervals of 20 minutes, to quantify $\mathrm{pH}$, lactate and base deficit ("biomarkers"). Fetal ECG recordings were collected with implanted electrodes and used to derive the fRR series.

Sample entropy (SampEn), permutation entropy (PE) and conditional PE ( $(P E)$ were estimated for fRR patterns of various lengths $m$, in each 2.5 minutes-long cycle of occlusion and successive recovery. Entropies' changes in time, during the course of the experiment, and their relation with the simultaneous values of the biomarkers were evaluated with Spearman's rank-order correlations.

Entropy values decreased during the experimental protocol $\left(r_{s}=-0.62\right.$ for SampEn at $m=1, r_{s}=-0.27$ for $P E$ at $m=5$ and $r_{s}=-0.30$ for $c P E$ at $m=4 ; p<0.05$ ). Correlations with biomarkers were found to be moderate for SampEn $\left(0.49<\left|r_{s}\right|<0.62 ; p<0.05\right)$ and weak to moderate for $P E$ and $C P E\left(0.31<\left|r_{s}\right|<0.54 ; p<0.05\right)$. Repetitive UCOs changed the regularity of $f R R$, suggesting a pronounced modulation as first line adaptive response.
\end{abstract}

\section{Introduction}

Labor exposes the fetus to repetitive transient hypoxic stress resulting from uterine contractions. Such contractions, that manifest with changes in fetal heart rate (FHR) variability through heart rate decelerations, are highly associated with acidemia [1], especially during the last 30 minutes before delivery. As a consequence, the fetus requires complex autonomic regulation to overcome the

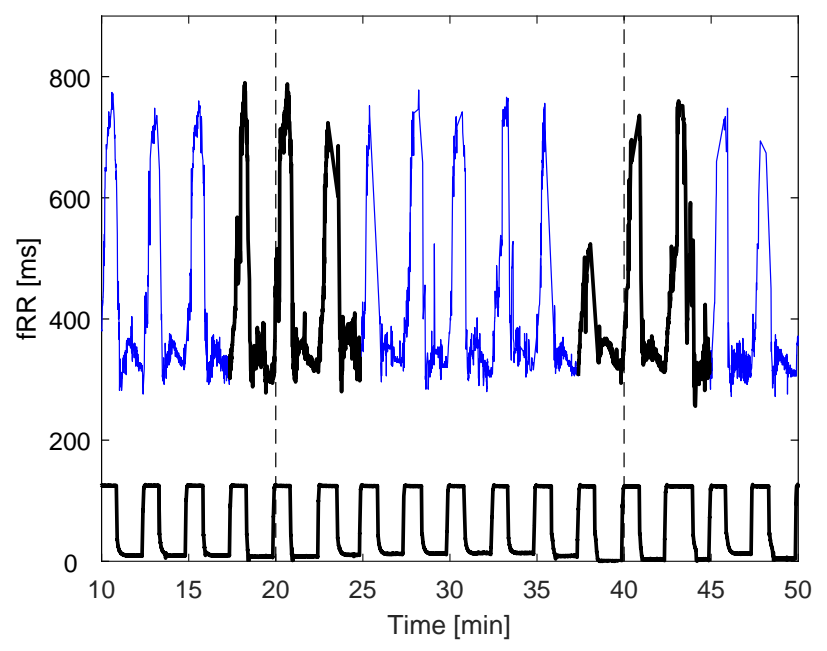

Figure 1. Example of Fetal RR series (filtered, TOP) overlapped to the UCO signal (BOTTOM, "SEVERE" occlusion). Dashed line indicates the blood sampling time. Bold black line shows the three occlusion-recovery periods closest to blood samples.

problem of hypoxia and acidemia. However, the precise role of the autonomic nervous system in regulating the fetal heart rate still remains not fully understood and needs further investigation. Indeed, the presence of acute hypoxia has been found to be associated with fluctuations in both sympathetic [2] and parasympathetic tones [3]. Such mechanisms are part of a complex interaction that involves several input signals such as chemoreceptor, baroreceptor and others. However, it is difficult to determine the exact interaction using standard heart rate variability analysis because of the high variability of its estimates [4].

In the context of heart rate variability analysis, signals' regularity has been quantified by means of many different entropy metrics. Sample Entropy (SampEn) [5] has been widely used for describing the effects of autonomic ner- 
vous regulations. The estimation of SampEn depends on the selection of two free parameters, i.e., the embedding dimension $m$ and the tolerance level $r$. Usually, $m$ is kept fixed to either 1 or 2 to avoid convergence issues (due to low number of samples) while the $r$ value is usually set as the $20 \%$ of the standard deviation of the RR series (as in this study). However, such tolerance value might become extremely large in presence of spikes or artifacts in the time series, thus altering the SampEn estimate.

Alternative approaches, not dependent on the tolerance value $r$, are Permutation Entropy (PE) and sorting entropy (also called conditional permutation entropy, cPE) [6]. PE is independent of the amplitude of the time series and is based on the ordering of patterns built with consecutive samples. This is quite useful for fRR analysis during labour, where artifacts and beat misdetections are common.

In this study, we verified whether the fetal autonomic regulation changes along with the progression of labor. To this aim, we employed a near-term pregnant sheep in-vivo model in which umbilical cord occlusions (UCOs) were instrumentally applied to mimic stress during labor. The autonomic regulation was quantified by means of three entropy measures, i.e., SampEn, PE and cPE, that were computed on the fetal inter-beat time interval series (fRR). Being PE and cPE independent of the actual values of the fRR samples, we speculated that they should better cope with the large variations in the local mean value induced by FHR decelerations, which were instead affecting the computation of acceleration and deceleration capacities (AC/DC) on the same dataset [7].

\section{Methods}

\subsection{Dataset and experimental protocol}

Fetal RR series collected from seven healthy near-term pregnant sheep were retrospectively analyzed in this study. Briefly, ewes and sheep fetuses were surgically instrumented with i) steel electrodes to measure the maternal and fetal electrocardiogram (fECG); and ii) inflatable silicon rubber cuff placed around the proximal portion of the umbilical cord to perform occlusions. Fetal ECG was then used to automatically derive the fRR series. Moreover, fetal arterial blood samples were collected at intervals of 20 minutes, to quantify three biomarkers related to lack of oxygen: $\mathrm{pH}$, lactate and base deficit. The dataset was previously described in [8].

UCOs were repetitively applied to the ovine fetus's umbilical cord. After a period of rest (BASELINE), a 1minute periodical mechanical compression continuously alternated with a 1.5 minutes recovery. Three levels of occlusion strength, from partial to complete, were designed: mild (MILD), moderate (MODERATE) and complete (SE-
VERE). The stimulation protocol lasted 1 hour for MILD and MODERATE, while for SEVERE, about $\sim 2$ hours or until $\mathrm{pH}<7.00$ was reached. As an example, figure 1 shows a portion of the fRR series of a single sheep during complete occlusion (SEVERE), as long as the timings of two blood samples. Further findings obtained using the same animal model can be found in $[7,8]$.

\subsection{Entropy measures}

Fetal RR series were analyzed by means of three entropy measures: SampEn, PE and cPE.

Briefly, SampEn [5] is an estimate of the entropy rate of a stochastic process (actually, the quadratic or Renyi entropy rate of order 2). Differently from SampEn, PE [6] measures the (Shannon) entropy of the ranks of the elements composing the $m$ dimensional vectors and it is widely used in biological time series [9] as well.

Finally, "sorting entropy", while defined along with PE in 2002 by Bandt \& Pompe, was largely overlooked until recently. It is often equivalently termed conditional permutation entropy (e.g., [10]), and we will follow this convention in here, for clarity. cPE determines the information contained in sorting the $m+1$ value among the previous $m$, when their order is already known (the estimate of an entropy rate):

$$
\mathrm{cPE}(\mathrm{m})=\mathrm{PE}(\mathrm{m}+1)-\mathrm{PE}(\mathrm{m}) .
$$

Both (empirical) PE and cPE provide upper bounds for the Kolmogorov-Sinai entropy [11], for $m \rightarrow \infty$.

In computing PE, tails were treated implicitly avoiding their existence by adding a small random number [6].

\subsection{Protocol of the study}

Two different analyses were performed. First, the time evolution of the entropy measures was assessed. For each 2.5 minutes-long cycle of occlusion and successive recovery (about 270 points, on average), SampEn, PE and cPE were estimated for patterns of various lengths ( $m=1$ to 3 for SampEn, $m=3$ to 6 for PE and $m=3$ to 5 for cPE). Second, we computed the same entropy measures on the three occlusion-recovery periods that were timealigned with each blood sample (from 1 cycle before to 1 after). Since biomarkers were slowly changing in time, we used three occlusion-recovery periods to increase the number of samples involved in the computation of entropy. The three occlusion-recovery periods employed for a given blood sample are exemplified in figure 1 .

\subsection{Statistical analysis}

Correspondingly to the two analyses described in the previous subsection, at first, we tested, for statistical sig- 

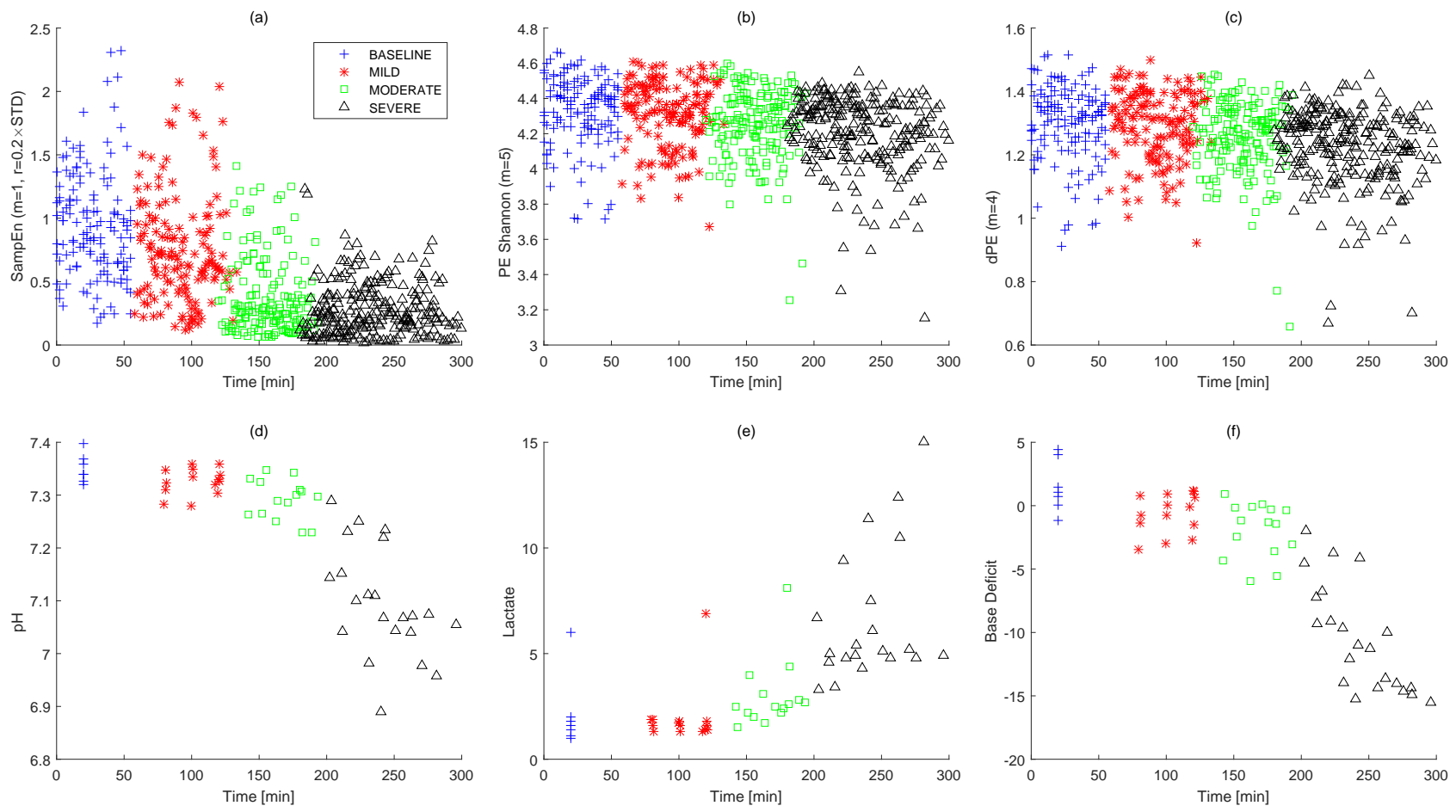

Figure 2. Time evolution of the three entropy measures (top panels) and biomarkers (bottom panels). Entropies are shown with different selections of the parameter $m$, i.e., $m=1$ for SampEn (a), $m=5$ for PE (b) and $m=4$ for cPE (c). pH, Lactate and Base Deficit are shown in panel (d), (e) and (f), respectively.

nificance, using the Spearman's rank-order correlation $r_{s}$ between entropy values and time, if they evolved during the course of the experiment. Secondly, we further verified the direct correlation between the values of the biomarkers and those of the entropies, computed on the three time aligned occlusion-recovery periods. A significance level of 0.05 was selected.

\section{Results}

Figure 2 shows the time evolution of the entropy measures for several $m$ values. As reported in Table 1, all entropy values decreased during the experimental protocol. In particular, SampEn displayed the highest correlation with time (e.g., $r_{s}=-0.62$ for SampEn at $m=2$ vs $r_{s}=-0.27$ for PE at $m=5$ and $r_{s}=-0.30$ for cPE at $m=4 ; p<0.05)$. Correlations with biomarkers were found to be moderate for SampEn $\left(0.49<\left|r_{s}\right|<0.62\right.$; $p<0.05)$ and weak to moderate for PE and cPE values $\left(0.31<\left|r_{s}\right|<0.54 ; p<0.05\right)$.

Since fRR during labor is corrupted by artifacts and gaps, we repeated the analysis after removing those fRR values which differed more than $20 \%$ from a running median of 11 samples. The results we obtained were equivalent and are not reported here. They nevertheless suggest that the limitations of permutation-based entropies high- lighted in [10] might not be validated by this study.

\section{Discussion \& Conclusions}

In this work, we studied the time evolution of the fetal autonomic regulation by means of entropy measures, in a in-vivo near-term healthy sheep model, mimicking labor.

SampEn, PE and cPE resulted in substantially similar correlations with biomarkers at different values of embedding dimension $m$. On the other hand, SampEn reported the highest correlation (i.e., change) with time. Such result might be partly due to the way we selected the tolerance value $(r=0.2 \times \mathrm{STD})$ and the presence of repetitive and periodical UCOs. Indeed, because of the increasing standard deviation of the fRR series during the protocol, due to the macro fRR oscillations, $r$ was also increased and SampEn affected. Fig. 3 reports the value of SampEn computed using $r=0.2 \times \mathrm{STD}$ at baseline (averaged over windows of 2.5 minutes). With a constant tolerance factor, SampEn now increased, coherently with the augmented RMSSD found in [3] (in fact, a larger spread of power across the spectral density leads to a larger differential entropy rate for any Gaussian process [12]).

Interestingly, the correlations we found were equivalently confirmed: i) using $\mathrm{PE}$ and $\mathrm{CPE}$, which are independent of the actual fRR sample's values; ii) after removing 
Table 1. Spearman's rank correlation of entropy estimates with time and biomarkers (pH, Lactate and Base Deficit), at different values of $\mathrm{m}$. Every correlation was significant $(p<0.05)$.

\begin{tabular}{lccc|cccc|ccc} 
& \multicolumn{3}{c}{ SampEn } & \multicolumn{3}{c}{ PE } & & \multicolumn{3}{c}{ cPE } \\
& $m=1$ & $m=2$ & $m=3$ & $m=3$ & $m=4$ & $m=5$ & $m=6$ & $m=3$ & $m=4$ & $m=5$ \\
\hline Time & -0.62 & -0.62 & -0.63 & -0.21 & -0.22 & -0.27 & -0.39 & -0.20 & -0.30 & -0.45 \\
pH & 0.62 & 0.59 & 0.60 & 0.47 & 0.45 & 0.40 & 0.42 & 0.39 & 0.31 & 0.39 \\
Lactate & -0.52 & -0.49 & -0.49 & -0.46 & -0.46 & -0.42 & -0.45 & -0.43 & -0.37 & -0.44 \\
Base Deficit & 0.62 & 0.60 & 0.61 & 0.54 & 0.48 & 0.43 & 0.43 & 0.41 & 0.37 & 0.34 \\
\hline
\end{tabular}

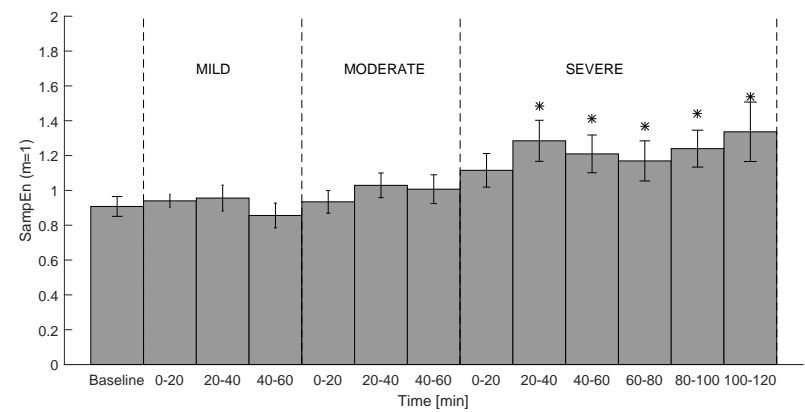

Figure 3. SampEn of fRR during the experimental protocol. Differently than Fig. 1 , here $\mathrm{m}=1$ and $\mathrm{r}=0.2 \times$ mean STD of baseline fRR. ${ }^{*} p<0.05$ versus baseline.

possible artifacts; and iii) even if the length of the time series analyzed was limited by the experimental protocol employed $(N \approx 270)$. In a future study, it would be interesting to verify if the changes detected by entropy metrics during the experimental protocol are indeed due to alterations of the nonlinear properties of the fRR series or if, instead, can be captured with a linear parametric model, as suggested in [12].

The results, confirming the findings obtained using acceleration and deceleration capacities [7], support the idea that repetitive umbilical cord occlusions led to a progressive change of regularity of the fetal HRV, suggesting a more pronounced FHR modulation, as first line adaptive response in healthy fetuses.

\section{References}

[1] Cahill AG, Roehl KA, Odibo AO, Macones GA. Association and prediction of neonatal acidemia. Am J Obstet Gynecol 2012;207:206.e1-206.e8.

[2] Siira SM, Ojala TH, Vahlberg TJ, Rosén KG, Ekholm EM. Do spectral bands of fetal heart rate variability associate with concomitant fetal scalp pH? Early Hum Dev 2013; 89:739-742.

[3] Durosier LD, Green G, Batkin I, Seely AJ, Ross MG,
Richardson BS, Frasch MG. Sampling rate of heart rate variability impacts the ability to detect acidemia in ovine fetuses near-term. Front Pediatr 2014;2:38.

[4] Koome ME, Bennet L, Booth LC, Davidson JO, Wassink G, Jan Gunn A. Ontogeny and control of the heart rate power spectrum in the last third of gestation in fetal sheep: Power spectral analysis of fetal heart rate. Exp Physiol 2014;99:80-88.

[5] Richman JS, Moorman JR. Physiological time-series analysis using approximate entropy and sample entropy. Am J Physiol Heart Circ Physiol 2000;278:H2039-H2049.

[6] Bandt C, Pompe B. Permutation Entropy: A Natural Complexity Measure for Time Series. Phys Rev Lett 2002; 88:174102.

[7] Rivolta MW, Stampalija T, Casati D, Richardson BS, Ross MG, Frasch MG, Bauer A, Ferrazzi E, Sassi R. Acceleration and Deceleration Capacity of Fetal Heart Rate in an In-Vivo Sheep Model. PLoS ONE 2014;9:e104193.

[8] Ross MG, Jessie M, Amaya K, Matushewski B, Durosier LD, Frasch MG, Richardson BS. Correlation of arterial fetal base deficit and lactate changes with severity of variable heart rate decelerations in the near-term ovine fetus. Am J Obstet Gynecol 2013;208:285.e1-6.

[9] Zanin M, Zunino L, Rosso OA, Papo D. Permutation Entropy and Its Main Biomedical and Econophysics Applications: A Review. Entropy 2012;14:1553-1577.

[10] Porta A, Bari V, Marchi A, Maria BD, Castiglioni P, di Rienzo M, Guzzetti S, Cividjian A, Quintin L. Limits of permutation based entropies in assessing complexity of short heart period variability. Physiol Meas 2015;36:755765.

[11] Unakafov AM, Keller K. Conditional entropy of ordinal patterns. Physica D 2014;269:94-102.

[12] Aktaruzzaman M, Sassi R. Parametric estimation of sample entropy in heart rate variability analysis. Biomed Signal Process Control 2014;14:141-147.

Address for correspondence:

Massimo Walter Rivolta

Via Bramante 65, Crema (CR), 26013, Italy

E-mail: massimo.rivolta@unimi.it 